\title{
Antitumor Effects of Dihydromyricetin on the Proliferation, Migration and Apoptosis of Human Hepatocellular Carcinoma Hep3B Cells
}

Lianggui Jiang

Guangdong Medical College

Wen-Chu Ye

Guangzhou Medical University

Zuobiao Li

Guangdong Medical College

Yongguang Yang

Guangdong Medical College

Wei Dai

Guangdong Medical College

Mingyi Li ( $\square$ limingyi63@163.com )

Guangdong Medical College

\section{Research Article}

Keywords: Hepatocellular carcinoma, Dihydromyricetin, Anti-tumor effect, Apoptosis

Posted Date: February 23rd, 2021

DOl: https://doi.org/10.21203/rs.3.rs-223378/v1

License: (9) (i) This work is licensed under a Creative Commons Attribution 4.0 International License.

Read Full License 


\section{Abstract}

Background: Hepatocellular carcinoma (HCC) represents a serious public health problem worldwide and has high morbidity and mortality. Dihydromyricetin (DHM) exhibits anti-tumor effect on a variety of malignancies, but its antitumor function of DHM in HCC has been unclear. The aim of this study was designed to investigate the antitumor effect of DHM on cell apoptosis, proliferation, migration and invasion of hepatoma carcinoma cells.

Methods: Cultured Hep3B cells were treated with different DHM concentrations, follow by cell apoptosis, proliferation, migration and invasion were examined by CCK-8, colony formation assay, wound healing, Transwell and flow cytometry, respectively. The mRNA and protein expression of apoptosis-associated genes and Bcl-2/Caspase-9 signaling pathway were validated by RT-PCR and western blot.

Results: DHM markedly suppressed proliferation, migration, invasion and facilitated apoptosis in Hep3B cells. Mechanistically, DHM significantly downregulated the Bcl-2 expression, and upregulated the mRNA and protein levels of Cleaved-Caspase 3, Cleaved-Caspase 9, Bak, Bax and Bad. Furthermore, in the nude mice tumorigenic model, DHM treatment greatly decreased the weight of the HCC tumors compared to the weights in control and NDP group.

Conclusions: DHM could suppress cell proliferation, migration, invasion, and facilitated apoptosis in Hep3B cells. These findings could provide novel insights to develop potential therapeutic strategy for the clinical treatment of HCC.

\section{Introduction}

Hepatocellular carcinoma (HCC) is the most common type of primary hepatocellular carcinoma and is a growing public health problem worldwide. Recently, the incidence, recurrence and mortality of HCC are continuously increasing over years in the majority of countries [1]. The increase in mortality mainly due to a lack of effective therapeutic options [2]. However, most treatments may cause serious side effects, such as nephrotoxicity, neurotoxicity and gastrointestinal (GI) toxicity [3]. Recent studies have illustrated that traditional Chinese medicines (TCMs) have benefic effects on the treatment of a variety of cancers, including HCC [4-6]. Therefore, to find a novel and effective TCM for the treatment of HCC, with the aim to improve the overall survival time of patients with HCC.

Dihydromyricetin (DHM), a biologically active flavonoid compound from Ampelopsis grossedentata [7], exerts anti-inflammatory, hypoglycemic, antioxidative, antimicrobial, anti-allergic, and anti-acne effects [8]. Remarkably, this flavonoid compound has attracted considerable attention because of its strong inhibitory effect on colorectal cancer [9], ovarian cancer [10], cholangiocarcinoma [11], and lung cancer [12]. It has been documented that DHM inhibited the progression of colorectal cancer and colon cancer in mouse models $[9,13]$. Besides, DHM also plays an important role in various biological processes including cell proliferation, apoptosis, and migration [14]. In vitro experiment demonstrated that DHM suppressed cell proliferation, migration, invasion and promoted apoptosis and cell cycle arrest at the 
G1/S phase in melanoma SK-MEL-28 cells, HCC [15], ovarian cancer cells [10], lung cancer cells [16], myelomonocytic lymphoma cells [17], and cholangiocarcinoma cells [14, 18].

In the present study, we mainly investigated the effect of DHM in the biological processes of cell growth and metastasis in Hep3B cells. The different concentrations of DHM were used to stimulate Hep3B cells to reveal the function of DHM on cell proliferation, apoptosis, migration and apoptosis. DHM stimulated Hep3B cells to reveal the anti-tumor characteristics both in vivo and in vitro. The apoptosis-associated genes and $\mathrm{Bcl}-2 /$ Caspase-9 signaling pathway were analyzed. Further, in the nude mice tumorigenic model, DHM treatment significantly reduced the weight of the HCC tumors. These findings might provide a potential therapeutic candidate for the clinical treatment of HCC.

\section{Materials And Methods}

\section{Cell culture and treatment}

Hep3B cells derived from American Type Culture Collection (ATCC, Rockville, MD, USA) were grown in Dulbecco's modified Eagle medium (DMEM, Life Technologies, Carlsbad, CA, USA) with $10 \%$ fetal bovine serum (FBS, Gibco, Australia). The cells were incubated in an incubator containing $95 \%$ air and $5 \% \mathrm{CO}_{2}$ at $37^{\circ} \mathrm{C}$. Dihydromyricetin (DHM) obtained from Sigma-Aldrich (St Louis, MO, USA) was dissolved in dimethylsulfoxide (DMSO, Sigma-Aldrich) and then different concentrations of DHM were used to stimulate Hep3B cells. Nedaplatin (NDP, Nichi-lko Pharmaceutical Co., Ltd.) was solubilized in sterilized H2O. DMSO served as a control group.

\section{Animals and tumor models}

The mice were housed under standard animal room conditions (temperature $22 \pm 1^{\circ} \mathrm{C}$ and humidity $55 \pm 5 \%$ ). Mice were anesthetized with $2 \%$ isoflurane (RWD, Shenzhen, China) using a Rodent Anesthesia Machine (VetEquip Inc., Pleasanton, $\mathrm{Ca}$ ). Animals were sacrificed with 2-3 times the anesthetic dose of isoflurane (via inhalation), followed by cervical dislocation, shaven and sterilized with $75 \%$ ethanol. After opening the abdominal cavity, the liver and tumor tissue were collected and weighed. All animals had free access to sterile tap water and food during the experiments. The mice were randomly divided into three groups, including control groups $(n=8), \operatorname{DHM}(n=8)$ and NDP $(n=8)$ for 3 weeks. Hep3B cells were transplanted into the mouse via subcutaneous injection of $1 \times 10^{7}$ cells. One week after transplantation, tumors had grown to a volume of approximately $20 \mathrm{~mm}^{3}$ with a model success rate of $100 \%$. All the animal experiments and surgical procedures were approved by the Institutional Animal Care and Use Committee of Guangdong Medical University (GDY1802018).

\section{Cell Viability Measurement}


The cell viability was assayed by adding Cell Counting Kit-8 assays (CCK-8) solution as described (CCK-8, Dojindo Molecular Technologies, Gaithersburg, MD)[19, 20]. The Hep3B cells were seeded in 6-well plates $\left(1 \times 10^{5}\right.$ cells/ well) and were allowed to adhere for $8 \mathrm{~h}$. The medium was replaced with medium containing different concentrations of $\operatorname{DHM}(0,10,20,30,40,50 \mu \mathrm{M})$. DMSO control wells contained $0.1 \%$ DMSO. After $12 \mathrm{~h}$, the culture medium of the cells was discarded, $10 \mu \mathrm{L}$ of CCK-8 solution was added into each well and cells were incubated at $37^{\circ} \mathrm{C}$ for another $2 \mathrm{~h}$. Finally, the absorbance was analyzed at 450 nm using a Microplate Reader (Molecular Devices, San Jose, CA, USA). All the assays were performed for three times independently. Absorbance of cells in the absence of treatment was regarded as $100 \%$ of cell survival. Cell survival was calculated as: absorbance/absorbance of control $\times 100 \%$.

\section{Colony formation assay}

Cell viability was performed using a colony formation assay [21]. Hep3B cells were seeded into a 6-well plate $\left(3 \times 10^{2}\right.$ cells/well) for $8 \mathrm{~h}$, followed by treatment with two different concentrations of DHM and NDP for $24 \mathrm{~h}$. Hep3B cells were cultured with drug-containing medium for ten days. The cells were fixed with methanol-glacial acetic acid stationary solution (3:1) at room temperature for $10 \mathrm{~min}$ and stained with $1 \%$ crystal violet (Amresco, Solon, OH, USA). The following formula was used to calculate the colony formation inhibition rate: Colony formation inhibition rate $=$ (control group -experimental group)/control group $\times 100 \%$.

\section{Cell apoptosis assay}

Cell apoptosis was assessed by flow cytometry assay (BD, FranklinLakes, NJ) [22]. In brief, the cells were seeded in 6-well plates $\left(1 \times 10^{6}\right.$ cells/well), followed by $24 \mathrm{~h}$ incubation at $37^{\circ} \mathrm{C}$. The cells were then treated with different concentrations of DHM and NDP for $24 \mathrm{~h}$. The assay was performed using the Annexin V-FITC/PI cell apoptosis detection kit (BD Pharmingen, USA) according to the manufacturer's protocol. Subsequently, the cells were monitored by flow cytometry (FACSCalibur, Becton Dickinson, USA), and the data were analyzed using FlowJo ${ }^{\mathrm{TM}}$ software (version 10, FlowJo LLC).

\section{Cell migration and invasion Assay}

Cell migration and invasion were detected by using Transwell assay with $8.0 \mu \mathrm{m}$ porous polycarbonate membranes (Millipore, Bedford, Massachusetts, USA) [20]. In brief, cells were treated with different concentration of DHM and NDP and adjusted cell density to $1 \times 10^{5}$. The lower transwell contained $600 \mu \mathrm{l}$ DMEM with $10 \% \mathrm{FBS}$. After incubation $24 \mathrm{~h}$ at $37^{\circ} \mathrm{C}$, the non-traversed cells in the upper compartment were wiped by a wet cotton swab. Traversed cells on the lower side of the filter were fixed stained with $0.1 \%$ crystal violet (Amresco, Solon, OH, USA). Then these cells were stained with $0.5 \%$ crystal violet (Merck, Darmstadt, Germany) for 20 min and counted microscopically (Olympus, Tokyo, Japan). The 
method of cell invasion was similar with cell migration, except that the inserts were coated with BD MatrigelTM Matrix (BD Biosciences, NY, USA).

\section{Wound healing assay}

Cell migratory abilities was tested by a wound healing assay. Hep3B cells were seeded in 12-well dishes $\left(5 \times 10^{4}\right.$ cells/well), and incubated in DMEM with $10 \% \mathrm{FBS}$ for $24 \mathrm{~h}$ at $37^{\circ} \mathrm{C}$. The cells were then exposed in the absence or presence of DHM and NDP. Then the cells were scratched across the surface of the well by pipette tip. After an incubation at $37^{\circ} \mathrm{C}$ of $24 \mathrm{~h}$, the scratches were observed.

\section{Western blot analysis}

The effects of DHM and NDP on the expression levels of Bcl-2, Cleaved-Caspase 3, Cleaved- Caspase 9, Bak, Bax and Bad were analyzed using western blot [20]. Protein samples were obtained from Hep3B cells that were treated with different concentrations of DHM and NDP for $24 \mathrm{~h}$ using cell lysis buffer (RIPA, Beyotime Biotechnology, Shanghai, China). The proteins were collected and detected by using the BCA ${ }^{\mathrm{Tm}}$ Protein Assay Kit (Pierce, Appleton, WI, USA). Subsequently, total protein $(20 \mu \mathrm{g})$ samples were separated using SDS-PAGE (10\% gel) and transferred onto a polyvinylidene fluoride (PVDF) membranes and blocked in $5 \%$ skim milk powder for $1 \mathrm{~h}$ at room temperature. Following the membranes were incubated with the corresponding antibodies. Primary antibodies of Bcl-2, Cleaved-Caspase 3, Cleaved-Caspase 9, Bak, Bax and Bad were (all from Cell Signaling Technology) were incubated with the membrane at $4{ }^{\circ} \mathrm{C}$ overnight. Then, blots were washed three times with TBST and were incubated with secondary antibodies for $1 \mathrm{~h}$ at room temperature. The blots were detected using enhanced chemiluminescence (ECL) reagents (Super Signal Dura kit, Pierce, IL, USA) according to the manufacturer's instructions. The blots were quantified by using Image Lab ${ }^{\text {TM }}$ Software (Bio-Rad).

\section{Hematoxylin and Eosin staining}

Briefly, liver tissues were immersed in $4 \%$ paraformaldehyde for $4 \mathrm{~h}$ and transferred to ethanol $(75 \%, 85 \%$, $95 \%)$. Then they were treated with xylene paraffin-embedded according to a previous report [23]. Before immunostaining, 3- $\mu \mathrm{m}$-thick liver tissue sections were dewaxed in xylene, rehydrated by decreasing concentrations of ethanol $(95 \%, 85 \%, 75 \%)$ and washed in PBS. And then stained with hematoxylin and eosin (H\&E). After staining sections were dehydrated through increasing concentrations of ethanol and xylene.

\section{Statements}

The study was approved by ARRIVE guidelines (http://www.nc3rs.org.uk/arrive-guidelines). Moreover, we confirm that all methods were performed in accordance with the relevant guidelines and regulations. 


\section{Statistical analysis}

All data are expressed as the mean \pm S.E.M., and at least three independent replicates were used for per group. All statistical procedures were analyzed by SPSS 22.0 (IBM, Manassas, VA, USA), and plots were generated using GraphPad prism 8.0 (GraphPad Software, La Jolla, CA, USA) (https://www.graphpad.com/scientific-software/prism/). The SPSS analysis show that our results are normal distribution, and homogeneity of results between each treatment groups are equal. Significant differences between treatment groups were determined by one-way ANOVA (SPSS 22.0, Chicago, IL, USA).

\section{Results}

\section{DHM suppressed cell proliferation and viability of Hep3B cells}

The Hep3B cells were cultured with different concentrations of DHM $(0 \mu \mathrm{M}, 10 \mu \mathrm{M}, 20 \mu \mathrm{M}, 30 \mu \mathrm{M}, 40 \mu \mathrm{M}$, $50 \mu \mathrm{M}$ for $12 \mathrm{~h}$, and then the cell proliferation was measured by CCK-8 assay. The results of the CCK-8 assay revealed that the proliferation of cells in the DHM group differed compared with the control group (Fig. 1A). The inhibitory effect increased prominently with increasing DHM concentration in a dose-dependent manner $(P<0.001)$ (Fig. 1A). In the subsequent experiments, 25 and $50 \mu \mathrm{M}$ of DHM were selected to treat Hep3B cells. According to the colony formation assay results (Fig. 1B and C), the cell viability in each group was significantly inhibited compared with the blank control group $(p<0.001)$, and the inhibition rate of 25 and $50 \mu \mathrm{M} \mathrm{DHM}(57.85 \% \pm 3.24 \%$; $88.55 \% \pm 0.759 \%)$ were higher compared with the same NDP $(33.64 \pm 2.73 \% ; 81.55 \% \pm 1.41 \%)$. These data indicated that DHM inhibited the viability and proliferation of Hep3B cells, and its effect was comparable to that of NDP.

\section{DHM inhibited the cell migration and invasion of Hep3B cells}

Cell migration and invasion of Hep3B cells were measured by Transwell assay in this study. The results of Transwell assay (Fig. 2A) illustrated that numerous cells migrated into the membrane of the upper chamber in the control group, whereas different doses of DHM and NDP treatment significantly inhibited the cell migration rate $(P<0.001)$ (Fig. 2A and 2B). Notably, the inhibitory effect on migration increased gradually with increasing DHM and NDP concentration in a dose-dependent manner $(P<0.001)$. The migration rate in the control group was obviously increased from that of the control group to $23.79 \pm$ $3.97 \%$ in the DHM $(25 \mu \mathrm{M}), 60.42 \pm 2.05 \%$ in the NDP $(25 \mu \mathrm{M}), 9.78 \pm 2.33 \%$ in the DHM $(50 \mu \mathrm{M})$ and $40.26 \pm 8.44 \%$ in the NDP $(50 \mu \mathrm{M})$ treatment groups (Fig. 2B). Furthermore, the wound healing assay revealed that DHM and NDP treatment significantly reduced wound closure rates in Hep3B cells (Fig. 2C and $\mathrm{D}$ ). In addition, compared with the control group, DHM and NDP treatment reduced the invasive ability of Hep3B cells (Fig. 2 C and D). These results suggested that DHM inhibited cell migration and 
invasion of Hep3B cells. Notably, the inhibition of migration and invasion in Hep3B cells following DHM treatment was superior to that by NDP treatment.

\section{DHM induced apoptosis in Hep3B cells}

As shown in Fig. 3A and B, the Hep3B cells were stained with FITC-Annexin-V and PI, and early and late apoptotic cells were measured by flow cytometry. The apoptosis rate of DHM and NDP group were significantly different from that of the control group $(P<0.001 ;$ Fig. 3B), and the apoptosis rate of the DHM group was also significantly different from that of the NDP group ( $P<0.001 ;$ Fig. $3 \mathrm{~A}$ and $3 \mathrm{~B})$. The proportion of apoptotic cells increased from $3.73 \pm 1.57 \%$ in the control group to $21.7 \pm 3.57 \%$ in the DHM $(25 \mu \mathrm{M}), 12.03 \pm 1.98 \%$ in the NDP $(25 \mu \mathrm{M}), 50.67 \pm 4.80 \%$ in the DHM $(50 \mu \mathrm{M})$ and $34.33 \pm 3.81 \%$ in the NDP $(50 \mu \mathrm{M})$ experimental groups following treatment for $12 \mathrm{~h}$ (Fig. 3B; $P<0.001$ versus control or NDP group). At $12 \mathrm{~h}$, apoptosis was higher $(P<0.001)$ in a dose-dependent manner of DHM-exposed Hep3B cells compared to those in NDP group. The percentages of early and late apoptotic cells significantly increased with an increase in drug concentration. Thus, DHM induced apoptosis of the Hep3B cells $(P<0.001)$, and the ability of DHM induce the apoptosis of Hep3B cells was better than NDP. Next, the protein levels of apoptosis-associated factors were measured by western blot. As displayed in Fig. 3C-3F, DHM and NDP significantly promoted cleaved caspase 3, cleaved Caspase 9, Bak, Bax and Bad expressions and inhibited Bcl-2 expression compared with control group $(P<0.001)$. These results suggested that DHM could induce apoptosis in Hep3B cells.

\section{Antitumor effects of DHM on tumor development in vivo}

The effect of DHM on the growth of primary tumor xenografts in nude mice was examined. Tumor volumes were recorded every three days. The volumes of the primary tumors in the DHM and NDP groups were greatly reduced compared with the control group, and the effect of the DHM treatment was superior to the effect of NDP (Fig. 4A). The weight of the tumors in the DHM group was only $0.26 \mathrm{~g}$ at the end of the experiment compared with the NDP group $(0.65 \mathrm{~g})$ and control group $(1.73 \mathrm{~g})$ (Fig. 4B, $P<0.001)$. These results illustrated that DHM exhibited the inhibition of tumor development. Liver tissues of nude mice were stained with HE (Fig. 4C). Hepatocytes in the DHM group were significantly enlarged, and could confirm the structure of hepatic lobules, which resulted in hepatic cords disordered and hepatic sinuses narrowed (Fig. 4C). However, DHM increased the weight of the mice compared to the NDP group (Fig. 4D), suggesting that DHM exhibited a better curative effect than NDP in suppressing the tumor development.

\section{Discussion}

At present, the treatment options of HCC mainly include orthotopic liver transplantation, surgical resection, local destruction, radiotherapy, and chemotherapy. Although there have been advances in the treatment of HCC patients, the worldwide recurrence and mortality rates of HCC and HCC-associated cases are very high. NDP is a broad-spectrum antitumor drug, and it may be used in the treatment of 
malignant tumors, such as cervical, nasopharyngeal, esophageal, and lung cancer [24-28]. In recent years, a number of studies have shown that the molecular mechanism of NDP in tumors, and this potentially involved multiple potential mechanisms. However, NDP treatment led to the autophagosome accumulation and increased LC3-II expression in cisplatin-sensitive nasopharyngeal cancer cell lines [29]. Furthermore, it has been demonstrated that high concentration of NDP could cause treatment-related side effects, such as nephrotoxicity, hematological toxicity, ototoxicity.

DHM, a naturally flavonoids of medicinal plants, has demonstrated therapeutic efficacy in the treatment of various cancer, and it has attracted attention as an antitumor agent against lung cancer, gastric cancer, ovarian cancer and liver cancer. DHM may be combined with or replace other chemotherapeutic drugs, such as NDP, in cancer therapy. Studying these molecular targets also provides novel theoretical foundation for understanding the molecular mechanisms of cancer, as well as novel drugs to replace NDP for cancer treatment. The safety of DHM has been studied in cell cultures, animals, healthy individuals and patients, and DHM is generally recognized as a safe extract of Rattan tea. In Hep3B cell culture studies, DHM inhibited cell proliferation and viability, migration, invasion, and promoted apoptosis. Furthermore, DHM treatment inhibited growth of xenotransplanted tumors in mice [30], suggesting the potential therapeutic effects of DHM as an antitumor agent.

The main antitumor mechanisms of DHM that have been described thus far are as follows: Inhibition of cell proliferation; induces apoptosis; inhibition of tumor metastasis. DHM significantly promoted pro-apoptotic protein expressions, such as cleaved caspase 3, cleaved Caspase 9, Bak, Bax and Bad expressions, but inhibited Bcl-2 expression, induce cell apoptosis of Hep3B cells. In addition, activation of the tumor suppressor gene p53 [10], and inhibition of Semaphorin 4D (Sema4D) [31], multidrug resistance protein 2 (MRP2) [32], NF-KB [33], and Notch1 pathway [15] and angiogenesis, can promote apoptosis and cytoprotective autophagy [33]. However, to the best of our knowledge, the antitumor effect of DHM in Hep3B cells has rarely been reported to date. The purpose of this study was to determine the effects of DHM on the proliferation, migration and apoptosis of Hep3B cells, implying that DHM may serve as a promising bioactive component for HCC treatment.

As is well known, caspases regulate cell proliferation and apoptosis [34]. Caspase family are usually divided into three protein categories: apoptosis initiators (caspase-9), apoptosis executioners (caspase-3 and -7 ) and inflammation mediators [35]. Previous research showed that caspase- 3 and caspase-9, are key apoptosis proteins in the apoptosis pathway [36]. The caspase-9 protein is the apoptosis initiator and the apoptosis executors (caspase-3 and -7) of cell apoptosis in mammals. The apoptosis initiator is first activated by apoptosis signals, followed by activation of apoptosis executioners of the downstream cascade. Ultimately, large amounts of substrates in cells are hydrolyzed for disintegration. Caspase-3 and caspase-9 are situated at vital junctions in apoptotic signaling pathways. Western blot analyses indicated that DHM treatment markedly promoted cleaved caspase 3, cleaved Caspase 9, Bak, Bax and Bad expressions, while inhibited Bcl-2 expression in Hep3B cells, which were consistent with other apoptosisrelated experiments in human myelomonocytic lymphoma cells [17]. Notably, DHM treatment matched or even exceeded the effect of NDP treatment on the caspase expression levels in Hep3B cells. 
Invasion and metastasis are key biological characteristics of malignant tumors. Adhesion molecules are involved in its malignant progression, invasion and metastasis. Cancer cells can invade stromal tissue the host stromal of the target organ by the blood vessel wall, which subsequently promotes tumor metastasis and invasion [37]. Transwell assays confirmed that DHM inhibited the migration and invasion of Hep3B cells in a dose-dependent manner, which was consistent with the results of Chen et al. reported that DHM reduced human cholangiocarcinoma cells migration and invasion [14]. Moreover, DHM treatment significantly reduced the weight of the HCC tumors. Thus, the data showed that the antitumor effects of DHM was better than that of NDP treatment.

\section{Conclusion}

These data demonstrated that DHM inhibited cell proliferation, migration, invasion, and promoted apoptosis of Hep3B cells. DHM may be critical for cell apoptosis and metastasis. The study hinted that DHM exhibited the anti-tumor effect on HCC, and might provide a novel sight into the clinical treatment of HCC. Further studies are still needed to uncover more potential effect of DHM on HCC.

\section{Abbreviations}

ANOVA: One-way analysis of variance; CCK-8: Cell Counting Kit-8; DHM: Dihydromyricetin; HCC: Hepatocellular carcinoma; TCMs: Traditional Chinese medicines; DMEM: Dulbecco's modified Eagle medium; DMSO: Dimethylsulfoxide; NDP: Nedaplatin; FBS: Fetal bovine serum

\section{Declarations}

\section{Acknowledgements}

Not applicable.

\section{Abbreviations}

ANOVA: One-way analysis of variance; CCK-8: Cell Counting Kit-8; DHM: Dihydromyricetin; HCC: Hepatocellular carcinoma; TCMs: Traditional Chinese medicines; DMEM: Dulbecco's modified Eagle medium; DMSO: Dimethylsulfoxide; NDP: Nedaplatin; FBS: Fetal bovine serum

\section{Funding}

This project was supported by National Natural Science Foundation of China (81041099), Guangdong Natural Sciences Foundation (S2011010003750) and Outstanding master's thesis cultivation project of Guangdong Medical University (2014-12). 


\section{Authors' contributions}

Mingyi li and Lianggui Jiang collected the data. Lianggui Jiang and Wen-Chu Ye conducted experiments and wrote the manuscript. Zuobiao LiهYongguang Yang and Wei Dai contributed to the discussion and reviewed the manuscript. All authors have read and approved the manuscript.

\section{Availability of data and materials}

All data and materials used in this current study are available from the corresponding author on reasonable request.

\section{Ethics approval and consent to participate}

All the animal experiments and surgical procedures were approved by the Institutional Animal Care and Use Committee of Guangdong Medical University (GDY1802018).

\section{Consent for publication}

Not applicable.

\section{Competing interests}

The authors declare that they have no conflicts of interest.

\section{References}

1. European Association for the Study of the Liver. Electronic address eee, European Association for the Study of the L: EASL Clinical Practice Guidelines: Management of hepatocellular carcinoma. $J$ Hepatol 2018, 69(1):182-236.

2. Tang L, Chen D, Guo L, Mo H, Huang Y, Guo S, Qi B, Tang Q, Wang P, Li X et al: Concurrent chemoradiotherapy with nedaplatin versus cisplatin in stage II-IVB nasopharyngeal carcinoma: an open-label, non-inferiority, randomised phase 3 trial. The Lancet Oncology 2018, 19(4):461-473.

3. Desoize $B$, Madoulet $C$ : Particular aspects of platinum compounds used at present in cancer treatment. Critical reviews in oncology/hematology 2002, 42(3):317-325.

4. Meng C, Bai C, Brown T, Hood L, Tian Q: Human Gut Microbiota and Gastrointestinal Cancer. Genomics, proteomics \& bioinformatics 2018, 16(1):33-49.

5. Wu P, Dugoua J, Eyawo O, Mills E: Traditional Chinese Medicines in the treatment of hepatocellular cancers: a systematic review and meta-analysis. Journal of experimental \& clinical cancer research : 
CR 2009, 28:112.

6. Liu X, Li M, Wang X, Dang Z, Yu L, Wang X, Jiang Y, Yang Z: Effects of adjuvant traditional Chinese medicine therapy on long-term survival in patients with hepatocellular carcinoma. Phytomedicine : international journal of phytotherapy and phytopharmacology 2019, 62:152930.

7. Zhang J, Chen Y, Luo H, Sun L, Xu M, Yu J, Zhou Q, Meng G, Yang S: Recent Update on the Pharmacological Effects and Mechanisms of Dihydromyricetin. Frontiers in pharmacology 2018, 9:1204.

8. Li H, Li Q, Liu Z, Yang K, Chen Z, Cheng Q, Wu L: The Versatile Effects of Dihydromyricetin in Health. Evidence-based complementary and alternative medicine : eCAM 2017, 2017:1053617.

9. Wang Z, Sun X, Feng Y, Wang Y, Zhang L, Wang Y, Fang Z, Azami N, Sun M, Li Q: Dihydromyricetin reverses MRP2-induced multidrug resistance by preventing NF-KB-Nrf2 signaling in colorectal cancer cell. Phytomedicine : international journal of phytotherapy and phytopharmacology 2020, 82:153414.

10. Xu Y, Wang S, Chan H, Lu H, Lin Z, He C, Chen M: Dihydromyricetin Induces Apoptosis and Reverses Drug Resistance in Ovarian Cancer Cells by p53-mediated Downregulation of Survivin. Scientific reports 2017, 7:46060.

11. Chen L, Yang Z, Zhou Y, Deng Y, Jiang P, Tan S: viaDihydromyricetin inhibits cell proliferation, migration, invasion and promotes apoptosis regulating miR-21 in Human Cholangiocarcinoma Cells. Journal of Cancer 2020, 11(19):5689-5699.

12. Kao S, Lee W, Chang J, Chow J, Chung C, Hung W, Chien M: Suppression of reactive oxygen speciesmediated ERK and JNK activation sensitizes dihydromyricetin-induced mitochondrial apoptosis in human non-small cell lung cancer. Environmental toxicology 2017, 32(4):1426-1438.

13. Zhu X, Lang H, Wang X, Hui S, Zhou M, Kang C, Yi L, Mi M, Zhang Y: Synergy between dihydromyricetin intervention and irinotecan chemotherapy delays the progression of colon cancer in mouse models. Food \& function 2019, 10(4):2040-2049.

14. Chen L, Yang Z, Zhou Y, Deng Y, Jiang P, Tan S: Dihydromyricetin inhibits cell proliferation, migration, invasion and promotes apoptosis regulating miR-21 in Human Cholangiocarcinoma Cells. Journal of Cancer 2020, 11(19):5689-5699.

15. Lu C, He Y, Yuan W, Xiang L, Zhang J, Liang Y, Duan J, He Y, Li M: Dihydromyricetin-mediated inhibition of the Notch1 pathway induces apoptosis in QGY7701 and HepG2 hepatoma cells. World journal of gastroenterology 2017, 23(34):6242-6251.

16. Fan $\mathrm{K}$, Yang B, Liu Y, Tian X, Wang B: Inhibition of human lung cancer proliferation through targeting stromal fibroblasts by dihydromyricetin. Molecular medicine reports 2017, 16(6):9758-9762.

17. Feng Q, Cui Z, Jin Y, Sun L, Li M, Zakki S, Zhou D, Inadera H: Protective effect of dihydromyricetin on hyperthermia-induced apoptosis in human myelomonocytic lymphoma cells. Apoptosis : an international journal on programmed cell death 2019, 24:290-300.

18. Zeng G, Liu J, Chen H, Liu B, Zhang Q, Li M, Zhu R: Dihydromyricetin induces cell cycle arrest and apoptosis in melanoma SK-MEL-28 cells. Oncology reports 2014, 31(6):2713-2719. 
19. Tamura K, Mawaribuchi S, Yoshimoto S, Shiba T, Takamatsu N, Ito M: Tumor necrosis factor-related apoptosis-inducing ligand 1 (TRAIL1) enhances the transition of red blood cells from the larval to adult type during metamorphosis in Xenopus. Blood 2010, 115(4):850-859.

20. Ai X, Mao F, Shen S, Shentu Y, Wang J, Lu S: Bexarotene inhibits the viability of non-small cell lung cancer cells via slc10a2/PPARY/PTEN/mTOR signaling pathway. BMC cancer 2018, 18(1):407.

21. Xiang L, He B, Liu Q, Hu D, Liao W, Li R, Peng X, Wang Q, Zhao G: Antitumor effects of curcumin on the proliferation, migration and apoptosis of human colorectal carcinoma HCT-116 cells. Oncology reports 2020, 44(5):1997-2008.

22. Xu Q, Tu J, Dou C, Zhang J, Yang L, Liu X, Lei K, Liu Z, Wang Y, Li L et al: HSP90 promotes cell glycolysis, proliferation and inhibits apoptosis by regulating PKM2 abundance via Thr-328 phosphorylation in hepatocellular carcinoma. Molecular cancer 2017, 16(1):178.

23. Tu S, Bhagat G, Cui G, Takaishi S, Kurt-Jones E, Rickman B, Betz K, Penz-Oesterreicher M, Bjorkdahl O, Fox $\mathrm{J}$ et al: Overexpression of interleukin-1 beta induces gastric inflammation and cancer and mobilizes myeloid-derived suppressor cells in mice. Cancer cell 2008, 14(5):408-419.

24. Blanchard $\mathrm{P}$, Tao Y: Nedaplatin in nasopharyngeal cancer: the rebirth of platinum salts? The Lancet Oncology 2018, 19(4):429-431.

25. Houl J, Ye Z, Brosey C, Balapiti-Modarage L, Namjoshi S, Bacolla A, Laverty D, Walker B, Pourfarjam Y, Warden $L$ et al: Selective small molecule PARG inhibitor causes replication fork stalling and cancer cell death. Nature communications 2019, 10(1):5654.

26. Tang F, Zhu J, Kong F, Ng M, Bian Q, Yam V, Tse A, Tse Y, Leung K: A BODIPY-based fluorescent sensor for the detection of Pt2+ and Pt drugs. Chemical communications (Cambridge, England) 2020, 56(18):2695-2698.

27. Li Y, Zeng J, Huang M, An J, Bai P, Wu L, Zhang R: A phase 2 study of nanoparticle albumin-bound paclitaxel plus nedaplatin for patients with advanced, recurrent, or metastatic cervical carcinoma. Cancer 2017, 123(3):420-425.

28. Ohnuma H, Sato Y, Hirakawa M, Okagawa Y, Osuga T, Hayashi T, Sato T, Miyanishi K, Kobune M, Takimoto R et al: A Phase 1/2 Study of Definitive Chemoradiation Therapy Using Docetaxel, Nedaplatin, and 5-Fluorouracil (DNF-R) for Esophageal Cancer. International journal of radiation oncology, biology, physics 2015, 93(2):382-390.

29. Liu Z, Liu J, Li L, Nie D, Tao Q, Wu J, Fan J, Lin C, Zhao S, Ju D: Inhibition of Autophagy Potentiated the Antitumor Effect of Nedaplatin in Cisplatin-Resistant Nasopharyngeal Carcinoma Cells. PloS one 2015, 10(8):e0135236.

30. Zhang Q, Liu J, Liu B, Xia J, Chen N, Chen X, Cao Y, Zhang C, Lu C, Li M et al: Dihydromyricetin promotes hepatocellular carcinoma regression via a p53 activation-dependent mechanism. Scientific reports 2014, 4:4628.

31. Liang J, Wu J, Wang F, Zhang P, Zhang X: Semaphoring 4D is required for the induction of antioxidant stress and anti-inflammatory effects of dihydromyricetin in colon cancer. International immunopharmacology 2019, 67:220-230. 
32. Wang Z, Sun X, Feng Y, Liu X, Zhou L, Sui H, Ji Q, E Q, Chen J, Wu L et al: Dihydromyricetin reverses MRP2-mediated MDR and enhances anticancer activity induced by oxaliplatin in colorectal cancer cells. Anti-cancer drugs 2017, 28(3):281-288.

33. Zhou D, Sun H, Yue J, Peng Y, Chen Y, Zhong Z: Dihydromyricetin induces apoptosis and cytoprotective autophagy through ROS-NF-KB signalling in human melanoma cells. Free radical research 2017, 51(5):517-528.

34. De Martino M, Vanpouille-Box C: Apoptotic Caspases: A Double-Edged Sword in Radiation-Induced Immunogenicity. Trends in cell biology 2019, 29(11):851-853.

35. Ma Y, Chang H: Caspase work model during pathogen infection. Virologica Sinica 2011, 26(6):366375.

36. Jiao C, Chen W, Tan X, Liang H, Li J, Yun H, He C, Chen J, Ma X, Xie Y et al: Ganoderma lucidum spore oil induces apoptosis of breast cancer cells in vitro and in vivo by activating caspase 3 and caspase9. Journal of ethnopharmacology 2020, 247:112256.

37. Hyoudou K, Nishikawa M, Umeyama Y, Kobayashi Y, Yamashita F, Hashida M: Inhibition of metastatic tumor growth in mouse lung by repeated administration of polyethylene glycolconjugated catalase: quantitative analysis with firefly luciferase-expressing melanoma cells. Clinical cancer research : an official journal of the American Association for Cancer Research 2004, 10(22):7685-7691.

\section{Figures}




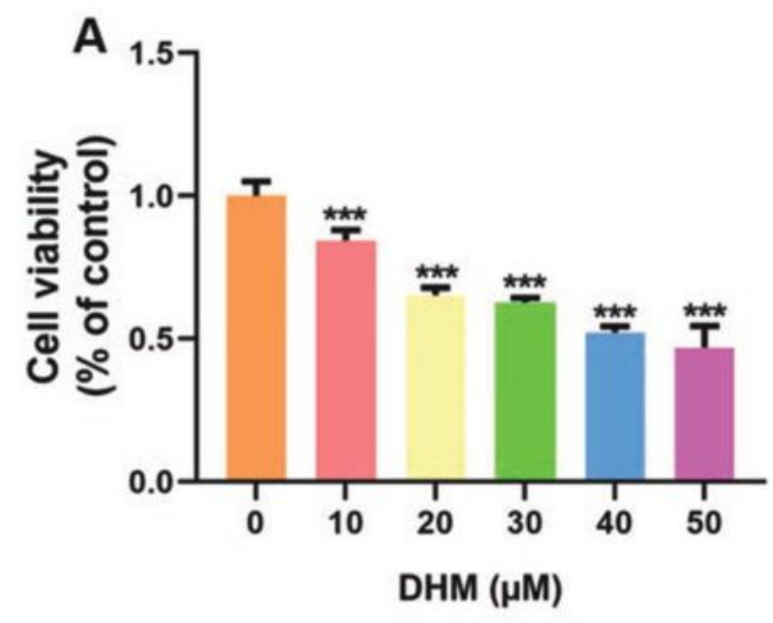

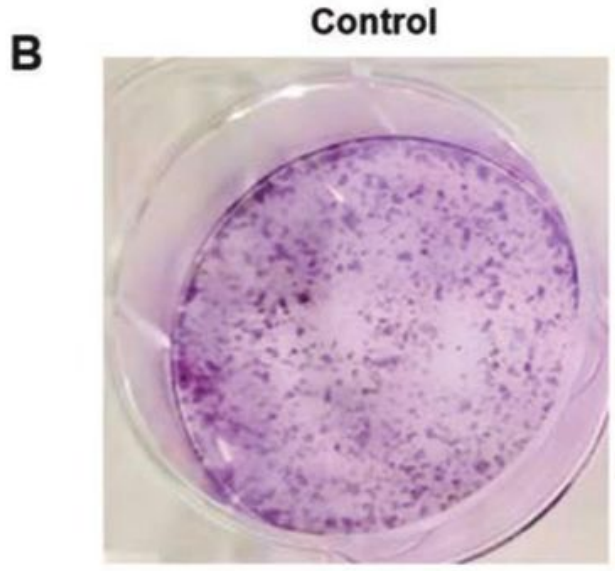

$50 \mu \mathrm{M}$ DHM

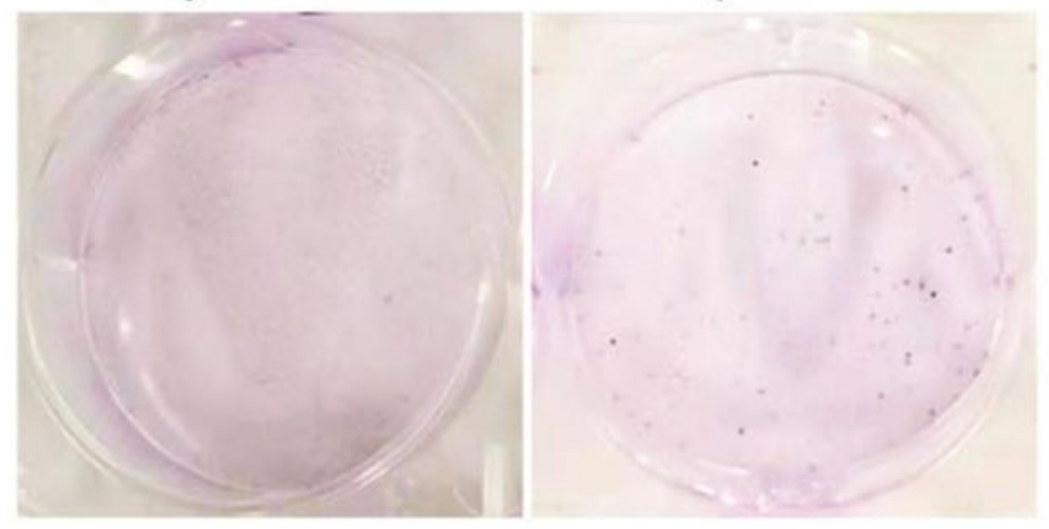

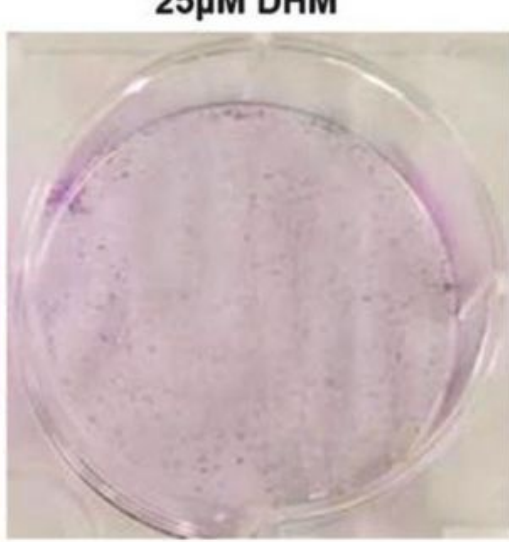

$50 \mu \mathrm{M}$ NDP
$25 \mu \mathrm{M}$ NDP

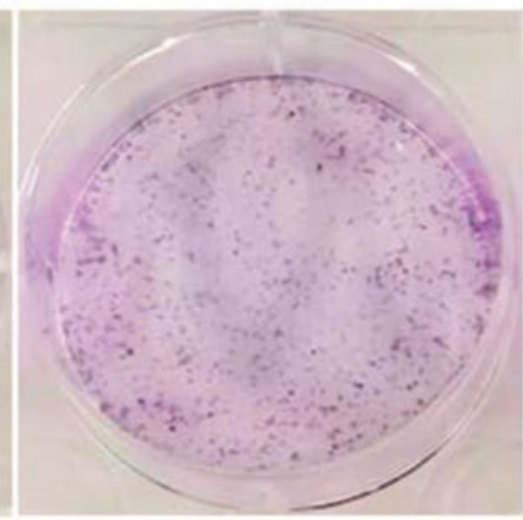

C

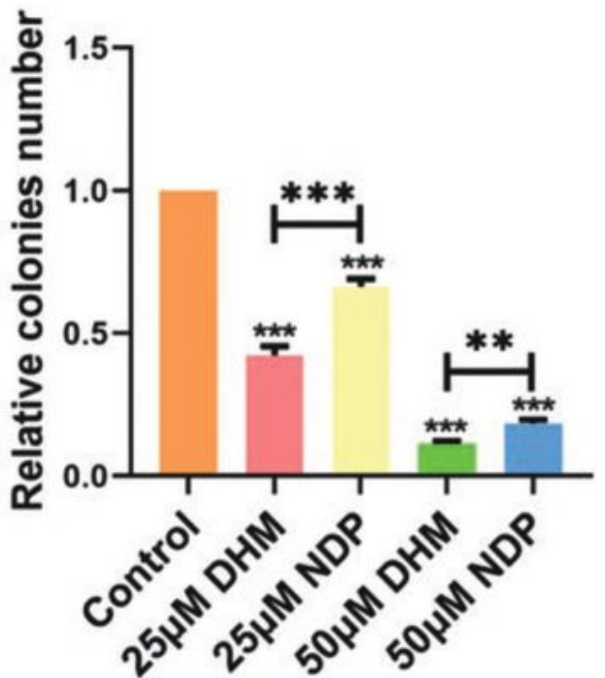

\section{Figure 1}

DHM inhibited cell proliferation and viability of Hep3B cells. (A) Hep3B cells were treated with six different doses of DHM $(0 \mu \mathrm{M}, 10 \mu \mathrm{M}, 20 \mu \mathrm{M}, 30 \mu \mathrm{M}, 40 \mu \mathrm{M}, 50 \mu \mathrm{M})$ of DHM for $12 \mathrm{~h}$. Cell proliferation was examined by CCK-8 assays. (B) Effects of DHM and NDP on viability of Hep3B cells according to a colony formation assay. (C) Quantification of colonies number in the colony formation assay. Results are 
presented as mean \pm standard error of mean $(n \geq 3)$. ${ }^{\star * P}<0.01$ and ${ }^{* \star *} P<0.001$ vs. control group or NDP group. NDP, Nedaplatin.

A

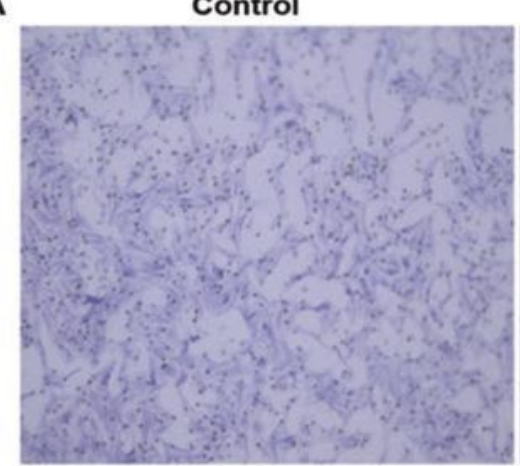

$25 \mu M$ NDP

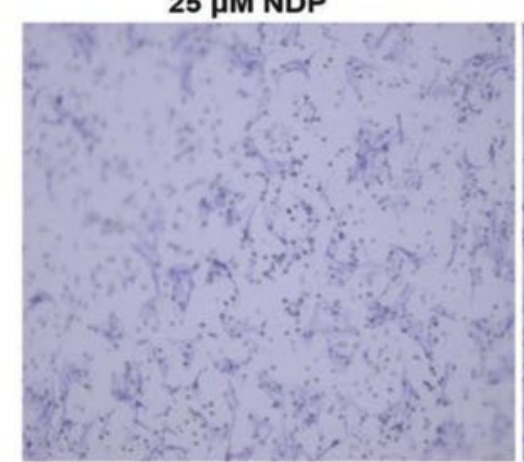

c

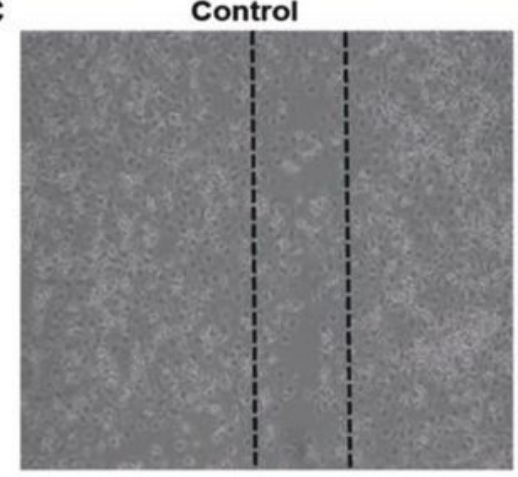

D

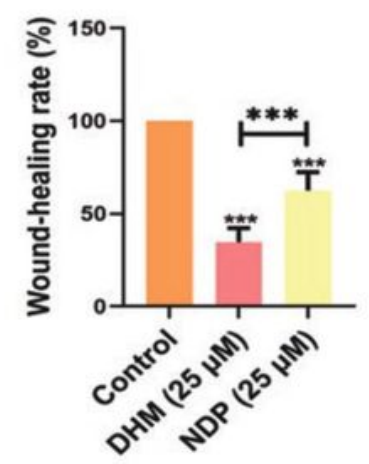

$25 \mu \mathrm{M}$ DHM

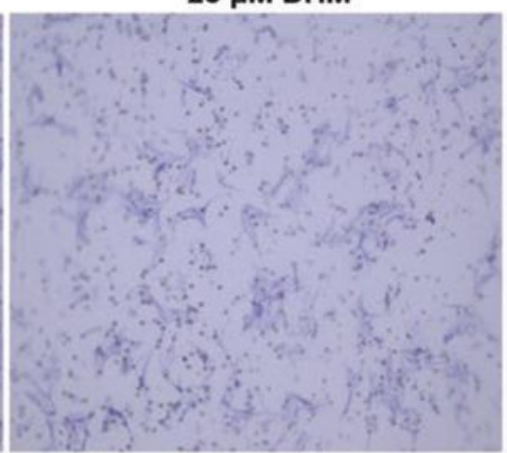

$50 \mu \mathrm{M}$ NDP

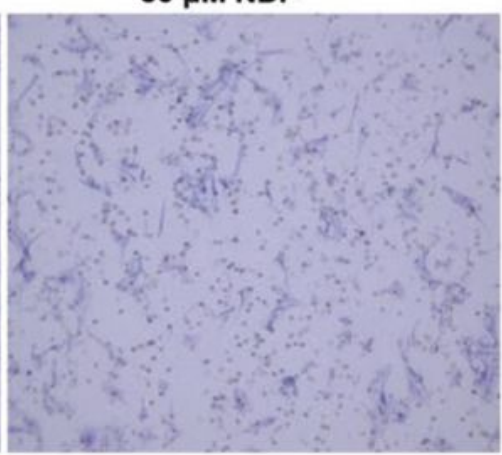

$25 \mu \mathrm{M}$ DHM
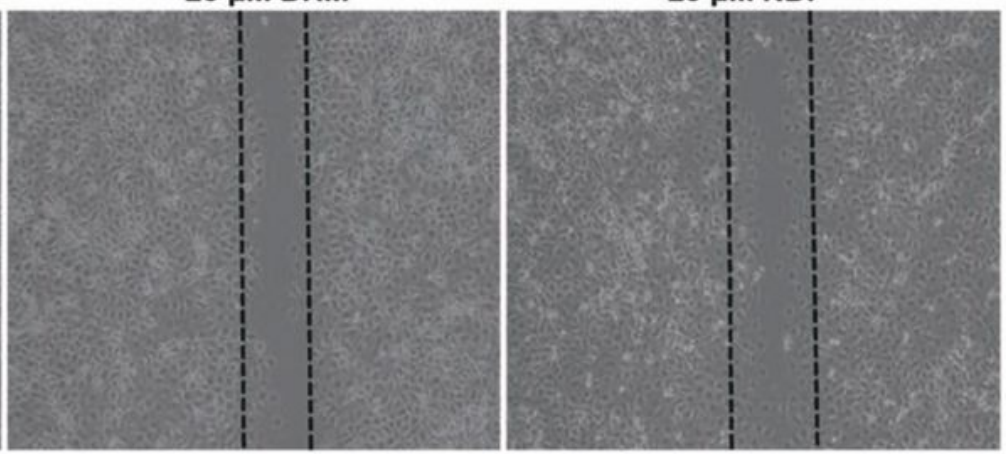

$50 \mu \mathrm{M}$ DHM

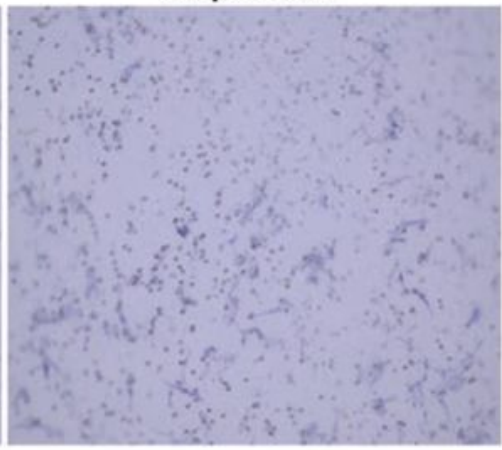

B

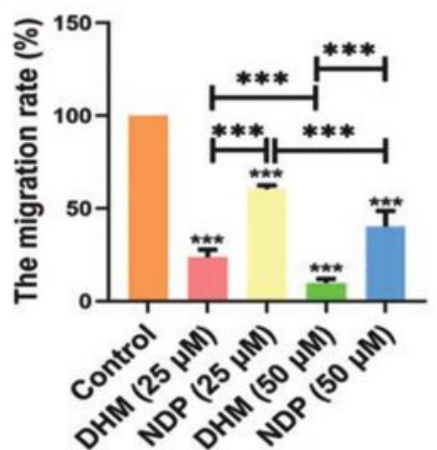

$25 \mu \mathrm{M}$ NDP

\section{Figure 2}

DHM inhibited cell migration and invasion of Hep3B cells. (A) Effect of DHM and NDP on the migratory ability of Hep3B cells in a Transwell assay. (B) Quantification of migration rate in the Transwell assay. Magnification, $x 200$. ${ }^{* *} P<0.001$. (C) Wound-healing assay. (D) Quantification of wound-healing rate in 
the wound healing assay. Results are presented as mean \pm standard error of mean $(n \geq 3)$. ${ }^{\star \star} P<0.01$ and $\star \star * P<0.001$ vs. control group or NDP group. NDP, Nedaplatin.
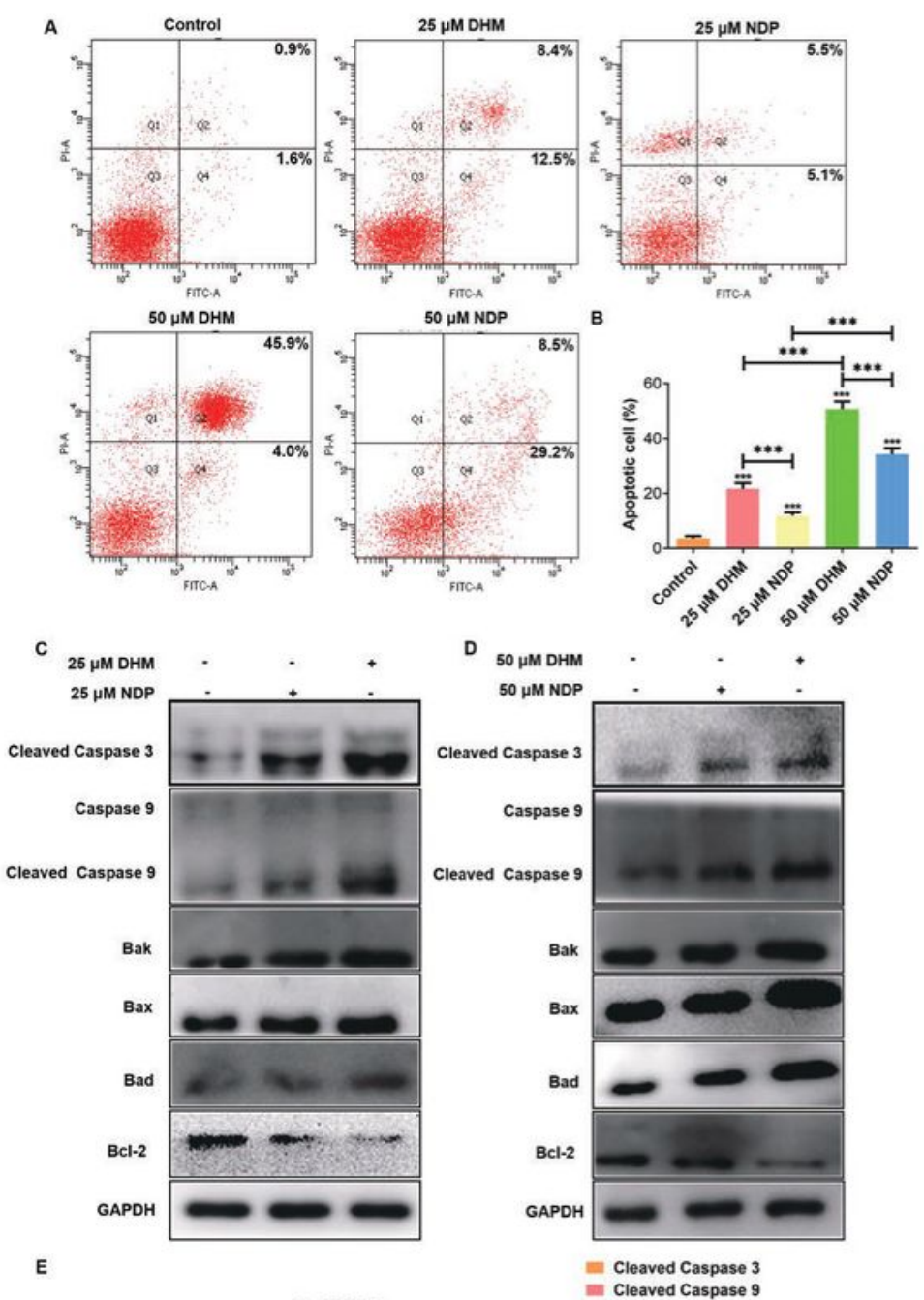

E
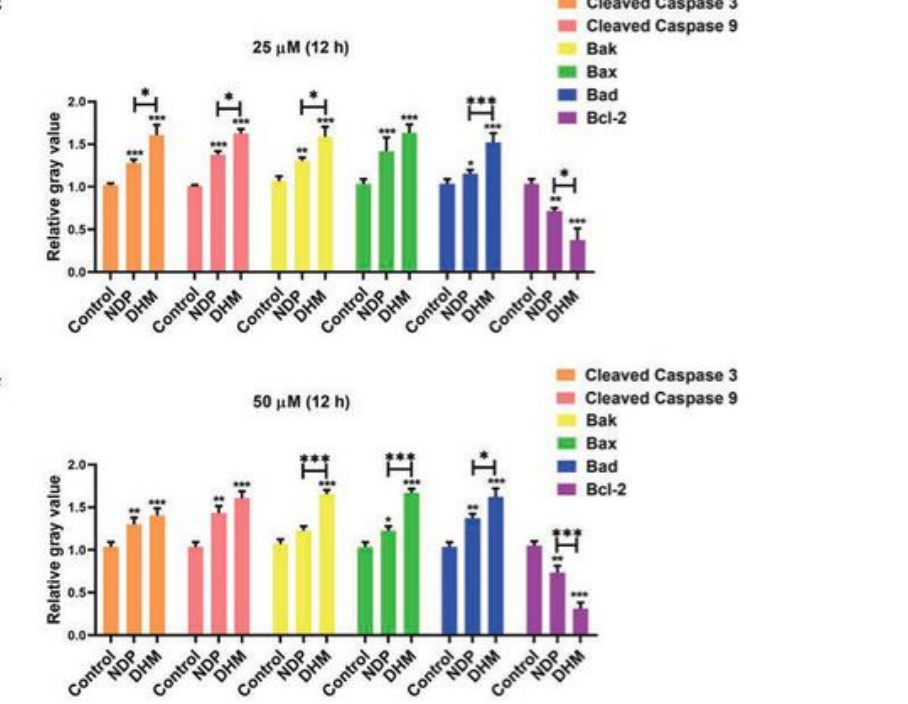

Figure 3

DHM induced apoptosis in Hep3B cells (A) Following treatment with five different group; Control, DHM ( 25 and $50 \mu \mathrm{M}$ ) and NDP (25 and $50 \mu \mathrm{M}$ ) for $12 \mathrm{~h}$, apoptosis rate of Hep3B cells was determined using Annexin V-FITC/PI dual-staining flow cytometry. (B) Quantification of apoptosis rate in Hep3B cells 
detected by Annexin V-FITC/PI dual-staining flow cytometry. (C and D) Apoptosis-associated factors (cleaved caspase 3, cleaved Caspase 9, Caspase 9, Bak, Bax, Bad and Bcl-2) were examined by western blot. Results are presented as mean \pm standard error of mean $(n \geq 3)$. ( $E$ and $F)$ Integrated density data were quantified. All images are representative of three independent experiments. NDP, Nedaplatin; $\mathrm{PI}$, propidium iodide. Data are means \pm SEM of three independent experiments, ${ }^{\star \star *} P<0.001 \mathrm{vs}$. control group or NDP group.

A

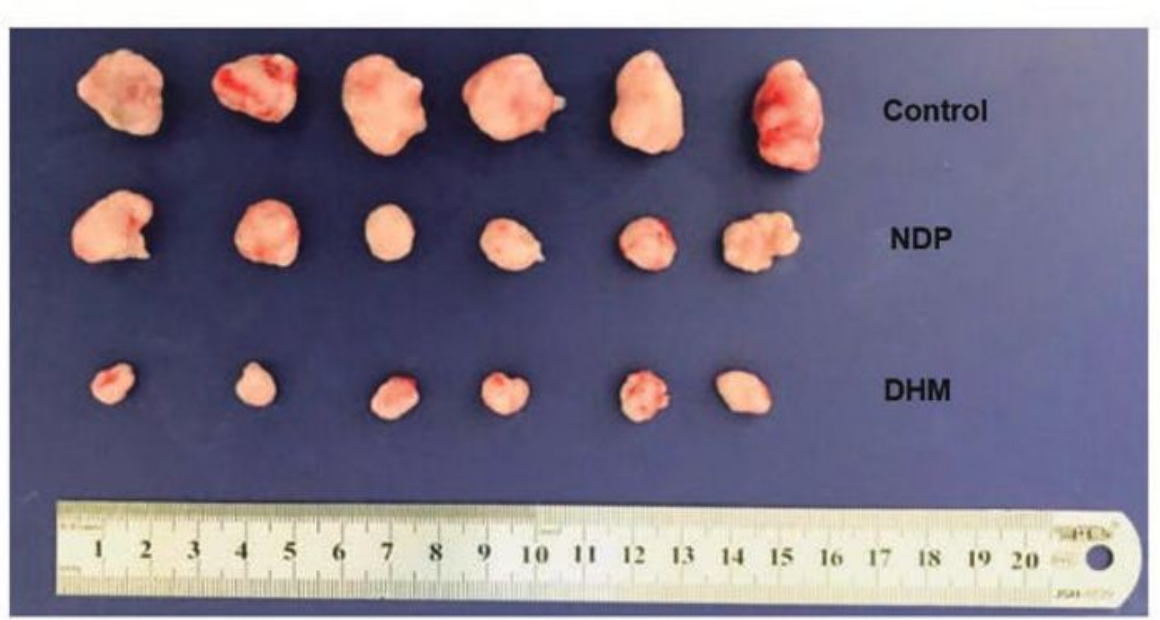

C
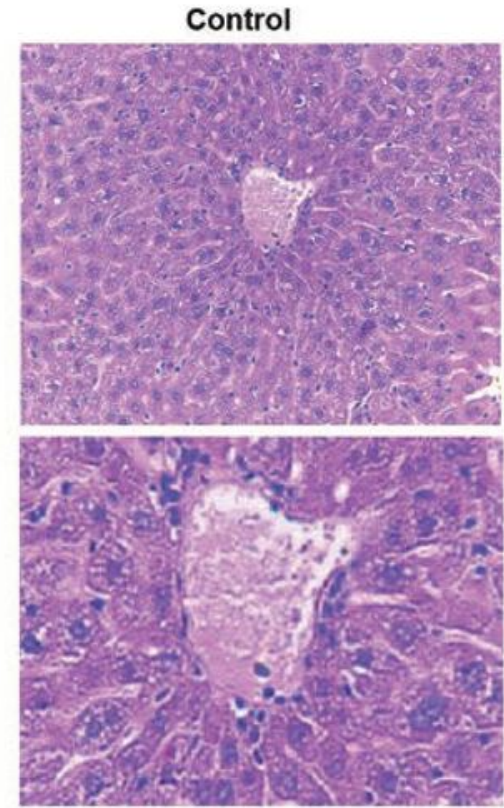

D
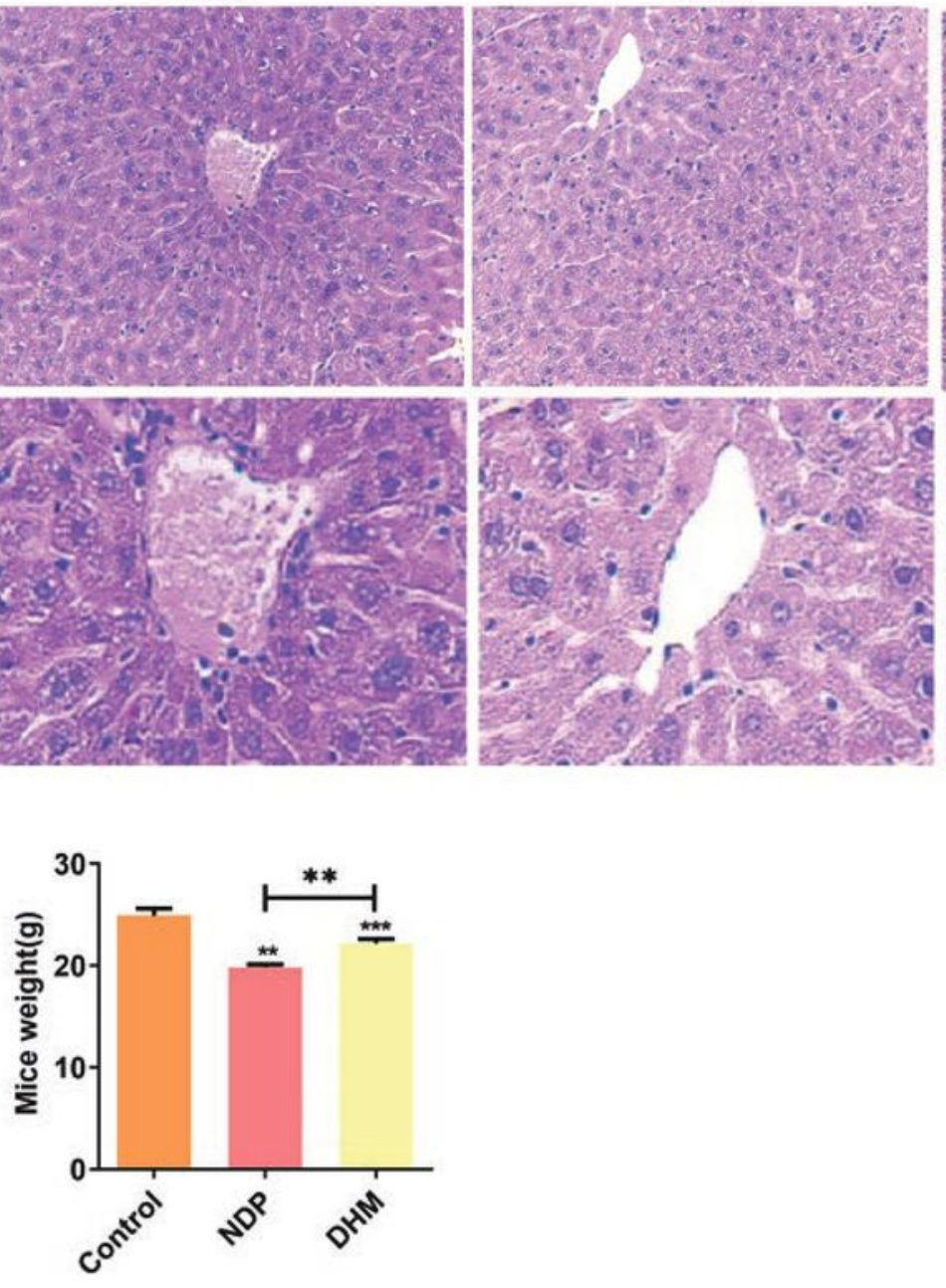

B

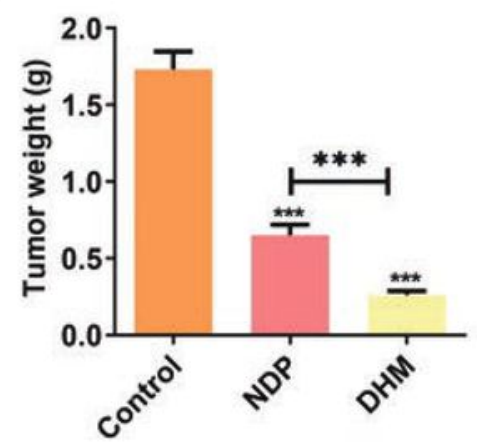

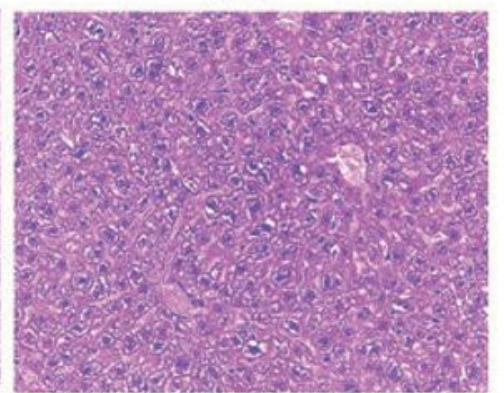

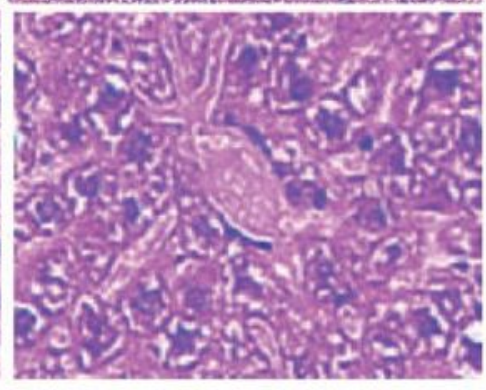

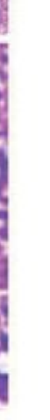


DHM inhibited tumor development in nude mice. (A) After nude mice were sacrificed, they were dissected to obtain tumors and photograph (lower). (B) Excised tumors were weighed separately. (C) Histologic analysis of HE staining of hepatic cords disordered and hepatic sinuses. (D) One week after last treatment, mice were sacrificed and weighed. Values are presented as the mean \pm SEM $(n=6) .{ }^{*}<<0.05$, ${ }^{\star *} \mathrm{P}<0.01$ and ${ }^{\star * *} \mathrm{P}<0.001$ compared with control or NDP group. 